\title{
Análisis Diagnóstico De Percepción En Adultos Jóvenes Sobre Participación Política Y Voto Electrónico En México
}

\author{
Irma Leticia Chávez-Márquez (PhD) \\ Liliana Álvarez-Loya (MARH) \\ María del Carmen Gutiérrez-Diez (PhD) \\ Oscar Alejandro Viramontes-Olivas (PhD) \\ Universidad Autónoma de Chihuahua, México
}

doi: 10.19044/esj.2017.v13n10p120 URL:http://dx.doi.org/10.19044/esj.2017.v13n10p120

\begin{abstract}
Mexico is challenging the perfection of democracy exercised by the people through voting. The objective of the research was to determine the feasibility of the exercise of electronic voting in Mexico, according to the political perception of young adults. The analysis is focused in the persons between 25 to 46 years old. This is a segment of the population of great importance within the economically active population in Mexico, and by the number of voters in this range, they have great influence on the results of the elections. The work was done from an analysis of the database obtained from the territorial survey conducted nationwide by the Center for Social and Political Studies (CESOP) of the LXII Legislature of the Chamber of Deputies in Mexico between November 21 and December 2, 2014; 1,400 interviews were conducted with 18 senior citizens spread over 132 municipalities throughout the country. The results were grouped into two variables, the first being the Political Participation with three indicators and the second, the Electronic Vote with six indicators. Cronbach Alfa Coefficient was obtained, with a value of 0.715.To measure the internal consistency of the research work, a multivariate factor analysis was performed. The main results indicate that they have low interest in politics. Their intention to vote is high on the following elections. The majority believes that electronic voting, people would come with greater certainty to cast the vote.
\end{abstract}

Keywords: Interest in politics, electoral processes, technology, voting, reliability 


\section{Resumen}

En México es un reto la perfección de la democracia ejercida por el pueblo mediante el sufragio. Por lo que el objetivo de este trabajo fue determinar la viabilidad del ejercicio del voto electrónico en México, de acuerdo a la percepción política de adultos jóvenes. El análisis comprende el rango de 25 a 46 años de edad. Este es un segmento poblacional de gran importancia dentro de la población económicamente activa, además por cuanto al número de votantes en este rango, tienen influencia en los resultados de comicios electorales. Se realizó un análisis de base de datos obtenida de encuestas territoriales aplicadas a nivel nacional por el Centro de Estudios Sociales y Políticos (CESOP) de la LXII Legislatura de la Cámara de Diputados en México del 21 de noviembre al 2 de diciembre de 2014; se realizaron 1,400 entrevistas a ciudadanos mayores de 18 años distribuidas en 132 municipios de todo el país. Los resultados fueron agrupados en dos variables, la primera es la Participación política con tres indicadores y la segunda, es el Voto electrónico con seis indicadores. Se obtuvo el Coeficiente Alfa de Cronbach, con valor de 0.715. Para medir la consistencia interna del trabajo, se desarrolló un análisis factorial multivariado. Los principales resultados indican que el interés en la política es bajo en la población; su intención de voto es alta respecto a las siguientes elecciones. La mayoría considera que con el voto electrónico, las personas acudirían con mayor certeza a emitir el sufragio.

Palabras clave: Interés en política, procesos electorales, tecnología, votación, confiabilidad

\section{Introducción}

El grado de participación política de los habitantes de un país, determina en gran medida el apoyo otorgado al trabajo realizado por los diferentes ámbitos de gobierno, además de generar certeza en la democracia. Las decisiones colectivas se toman mediante elecciones y plebiscitos, por lo que es relevante el cómo los ciudadanos participan en la vida política de un país (Holzner, 2007). En México votan menos los ciudadanos de bajos recursos económicos que los de posición media o alta.. El voto se ha visto como un arma política y no sólo como un derecho en México, sin embargo la desinformación a la hora de emitirlo, no fortalece una adecuada rendición de cuentas (Hernández, 2008). La aplicación del voto electrónico, se trata de un gran y profundo cambio que generará diferentes impactos de orden social, político y jurídico; no es sólo la incorporación el día de los comicios de equipos electrónicos (Tula, 2012). Los contratiempos registrados con máquinas de votar pueden ser mínimos, a pesar de que el voto electrónico genere desconfianza (Velázquez Trujillo, 2010). Históricamente, en México 
ha sido un reto la perfección de la democracia ejercida por el pueblo mediante el voto, donde se han realizado observaciones de prácticas que han generado cada vez más un mayor control sobre el voto ejercido. El objetivo del trabajo fue determinar la viabilidad del ejercicio del voto electrónico en México, de acuerdo a la percepción política de adultos jóvenes entre 25 y 46 años.

\section{Materiales y métodos}

El trabajo se realizó a partir de un análisis de la base de datos obtenida de la encuesta territorial aplicada a nivel nacional por el Centro de Estudios Sociales y Políticos (CESOP) de la LXII Legislatura de la Cámara de Diputados en México entre el 21 de noviembre y el 2 de diciembre de 2014; se realizaron 1,400 entrevistas a ciudadanos mayores de 18 años distribuidas en 132 municipios de todo el país. A partir de esta encuesta, se analizó la base de datos y se observa que un alto porcentaje de votantes (45\%) están comprendidos entre los 26 y 45 años de edad; siendo estos en los cuales se centra el análisis estadístico presentado en este trabajo de investigación.

Del total de la muestra, 679 se encuentran en el rango de 26 a 45 años de edad, de los cuales 352 son del sexo femenino y 327 del masculino, con un $51.84 \%$ y $48.15 \%$ respectivamente del total del rango considerado para este trabajo. En el (Cuadro 1) se desglosa el número de votantes por género.

Cuadro 1. Número de votantes adultos jóvenes, por género y edad

\begin{tabular}{|c|c|c|c|c|c|c|c|c|c|}
\hline \multirow[t]{2}{*}{ Edad } & \multicolumn{2}{|l|}{ Sexo } & \multirow[t]{2}{*}{ Total } & \multirow[t]{2}{*}{$\%$} & \multirow[t]{2}{*}{ Edad } & \multicolumn{2}{|c|}{ Sexo } & \multirow[t]{2}{*}{ Total } & \multirow[t]{2}{*}{$\%$} \\
\hline & $\mathrm{F}$ & $M$ & & & & $\mathrm{~F}$ & $\mathrm{M}$ & & \\
\hline 26 & 17 & 18 & 35 & 5.15 & 36 & 7 & 9 & 16 & 2.35 \\
\hline 27 & 21 & 15 & 36 & 5.30 & 37 & 2 & 12 & 14 & 2.06 \\
\hline 28 & 26 & 12 & 38 & 5.59 & 38 & 18 & 9 & 27 & 3.97 \\
\hline 29 & 9 & 8 & 17 & 2.50 & 39 & 22 & 16 & 38 & 5.59 \\
\hline 30 & 18 & 27 & 45 & 6.62 & 40 & 35 & 42 & 77 & 27.59 \\
\hline 31 & 10 & 10 & 20 & 2.94 & 41 & 12 & 10 & 22 & 7.88 \\
\hline 32 & 25 & 10 & 35 & 5.15 & 42 & 15 & 24 & 39 & 5.74 \\
\hline 33 & 15 & 16 & 31 & 4.56 & 43 & 15 & 14 & 29 & 4.27 \\
\hline 34 & 18 & 16 & 34 & 5.00 & 44 & 19 & 16 & 35 & 5.15 \\
\hline 35 & 9 & 12 & 21 & 3.09 & 45 & 39 & 31 & 70 & 10.3 \\
\hline
\end{tabular}

En la muestra de la encuesta aplicada, la mediana de votantes por edad es de 37, lo cual indica que una mitad es menor de 37 años y la otra es mayor a esa edad. Este valor de la mediana, queda dentro del rango de edad analizado en esta investigación. El procesamiento de los datos se realizó mediante el programa Excel de Microsoft Office y el estadístico SPSS. 


\section{Resultados y discusión}

Los resultados se presentan agrupados en dos variables, ya que se trabajó la relación entre Participación política e Implementación del voto electrónico. Se puede considerar la implementación del voto electrónico, sólo como un instrumento de la administración electoral que puede ayudar a mejorar la calidad de la democracia, si el contexto y las condiciones del país en donde se aplique son las adecuadas al potencial que ofrecen las nuevas tecnologías (Barrientos, 2007).

La variable Participación política se midió mediante tres indicadores: Interés en la política; Probabilidad de acudir a votar e Interés en los procesos electorales. En segundo término, se presentan la variable Implementación del voto electrónico, medida con seis indicadores: Influencia de la tecnología en la confiabilidad del voto electrónico; Tipo de tecnología para emitir el voto electrónico; Grado de confiabilidad con el voto electrónico; Mayor votación con voto electrónico; Costos del voto electrónico y finalmente Urgencia de implementación del voto electrónico.

Se obtuvo el Coeficiente Alfa de Cronbach como validación del instrumento de medición para los nueve indicadores considerados, arrojando un valor confirmatorio como se muestra en el (Cuadro 2).

Cuadro 2. Estadísticos de fiabilidad

\begin{tabular}{ccc}
\hline Alfa de Cronbach & $\begin{array}{c}\text { Alfa de Cronbach basado en los } \\
\text { elementos tipificados }\end{array}$ & Número de elementos \\
\hline 0.693 & 0.715 & 9 \\
\hline
\end{tabular}

Para medir la consistencia interna del trabajo de investigación, se realizó un análisis factorial multivariado, para confirmar la agrupación de indicadores para las dos variables consideradas, Participación política e Implementación del voto electrónico; con tres y seis indicadores respectivamente. Una técnica utilizada para descubrir agrupaciones de indicadores de manera que los indicadores de cada grupo están altamente correlacionados, es el análisis factorial y además los grupos de indicadores son relativamente independientes. De esta manera se consigue reducir un número de indicadores intercorrelacionados a un número inferior de factores no correlacionados, lo cual permite explicar la mayor parte de variabilidad de cada una de las variables (Montoya, 2007). Con el análisis factorial multivariado, se obtuvo la agrupación de los indicadores en los dos constructos de variables analizados con el Método de Extracción, obteniendo dos componentes, uno para cada variable; lo cual se muestra en el Cuadro 3. 
Cuadro 3. Matriz de componentes con el Método de Extracción

\begin{tabular}{ccc}
\hline Variable & Componente & \\
\cline { 2 - 3 } & 1 & 0.660 \\
Interés en la política & & 0.648 \\
Probabilidad de acudir a votar & & 0.674 \\
Interés en los procesos electorales & 0.525 & \\
$\quad$ electrónico & 0.705 & \\
Influencia de la tecnología en la confiabilidad del voto & 0.631 & \\
Tipo de tecnología para emitir el voto electrónico & 0.729 & \\
Grado de confiabilidad con el voto electrónico & 0.672 & \\
Mayor votación con voto electrónico & 0.608 & \\
Costos del voto electrónico & & \\
Urgencia de implementación del voto electrónico & &
\end{tabular}

Se obtuvo también la medida de adecuación muestral de KaiserMeyer-Olkin (KMO), para demostrar si el instrumento de medición tiene consistencia interna y si es significativo, arrojando un excelente resultado, ya que es mucho mayor al mínimo aceptable de 0.5; además con la prueba de esfericidad de Bartlett, se obtuvo la Chi cuadrada que arrojó un valor que confirma junto con el nivel de significancia de 0.000; con lo cual se puede observar que sí están relacionadas las variables; ambas pruebas se muestran a continuación en el (Cuadro 4).

Cuadro 4. Medida KMO y Prueba de Bartlett para los constructos de participación política y voto electrónico

$\begin{array}{ccc}\text { Medida de adecuación muestral de } & \text { KMO } & 0.815 \\ \text { Kaiser-Meyer-Olkin } & & \\ \text { Prueba de esfericidad de Bartlett } & \text { Chi-cuadrado aproximado } & 1320.933 \\ & \text { gl } & 45 \\ & \text { Significancia } & .000\end{array}$

\section{Participación política}

$\mathrm{Al}$ abordar el tema de interés en la política, 38\% indica que no le interesa nada; $37 \%$ que le interesa poco, en suma, al $75 \%$ le interesa la política en muy bajo grado, lo cual, puede reflejarse en una baja participación al momento de votar. Se mostran los resultados en la (Gráfica $1)$. 
Gráfica 1. Interés en la política

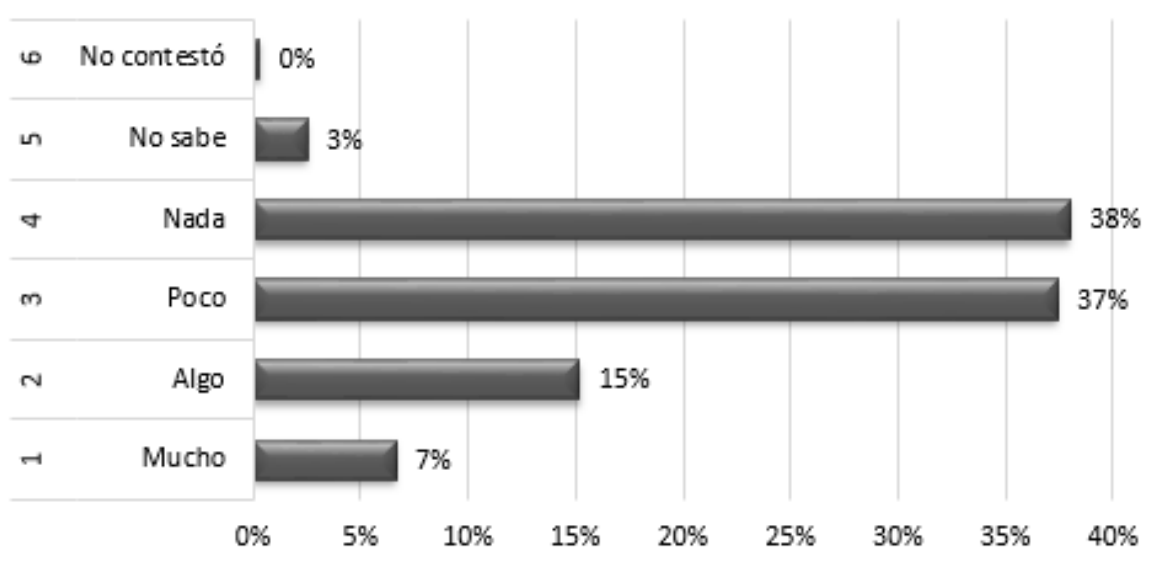

La democracia en México no ha estado a la altura de las expectativas de muchos ciudadanos, tampoco ha generado más igualdad social ni económica; pocas personas creen en la actualidad que su participación política sea más efectiva que en el pasado; muy frecuentemente el gobierno elegido, no responde a las demandas de los ciudadanos.

Los mexicanos están insatisfechos con su gobierno democrático, en gran parte porque no les ha provisto de los bienes sociales que a menudo esperan de la democracia, realista o no esta perspectiva. También existe descontento debido a la brecha que perciben entre su concepción idealizada de la democracia y la forma de operar de las instituciones democráticas existentes. Existe cierta evidencia de que no alcanzar la equidad es una de las fuentes de insatisfacción con la democracia entre la ciudadanía (Aguilar, 2014).

Respecto al evento de acudir a votar, $30 \%$ manifiesta que definitivamente sí votará y $27 \%$ que probablemente, con lo cual, se puede esperar que $57 \%$ de la población es la que tiene mayor probabilidad de emitir su voto, sin embargo continúa siendo muy alto el porcentaje de personas que probablemente no acudirá a sufragar. Mostrándose en la (Gráfica 2). 
Gráfica 2. Probabilidad de acudir a votar en las próximas elecciones

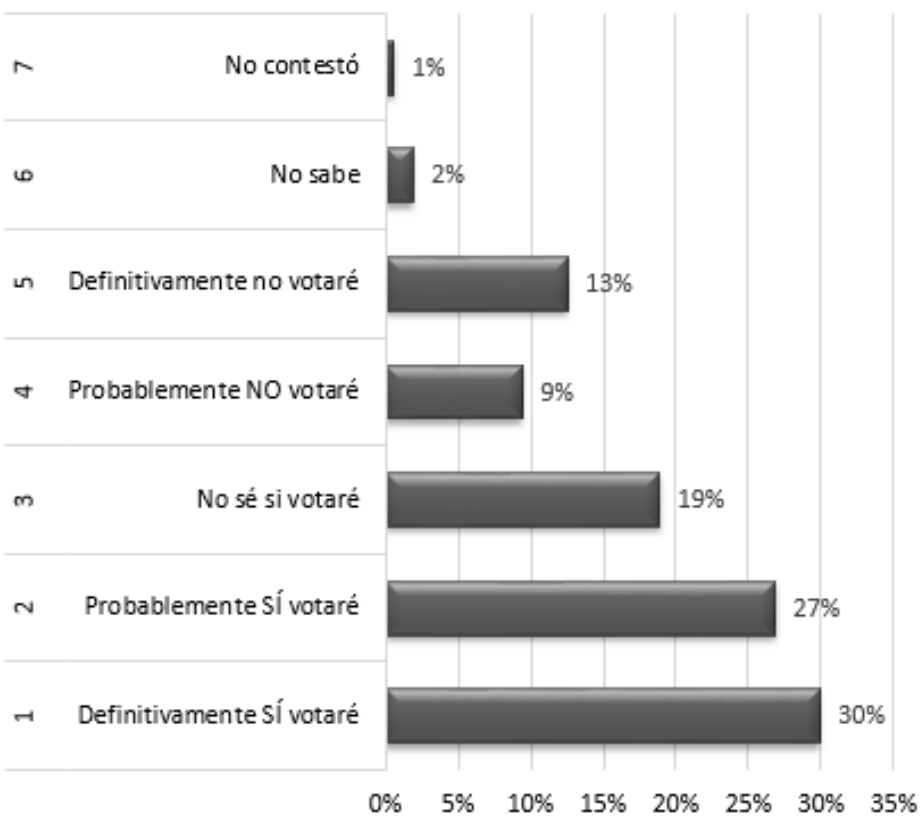

El hecho de considerar la posibilidad de no ejercer el derecho de emitir el sufragio, puede deberse a la percepción de la falta de equidad, que se mezcla con otras causas de insatisfacción. Es difícil establecer cuál es el impacto específico y las consecuencias políticas del discurso de la equidad.

Es importante subrayar que este argumento tiene claras limitaciones: sólo se propone que éste es uno de los factores que explican el malestar generalizado con la democracia. No se pretende determinar qué tanto ha contribuido la noción de equidad al descontento ciudadano. Con la información disponible no es posible dar cuenta con precisión del peso específico de este factor (Aguilar, 2014).

$\mathrm{Al}$ analizar el interés en los procesos electorales por parte de los votantes, se obtiene que un $59 \%$ de la población es la que se encuentra muy, poco o algo interesada en los procesos electorales, como lo muestra la (Gráfica 3), por lo cual se puede relacionar con el hecho de un alto grado de abstencionismo en México. 
Gráfica 3. Interés en los procesos electorales

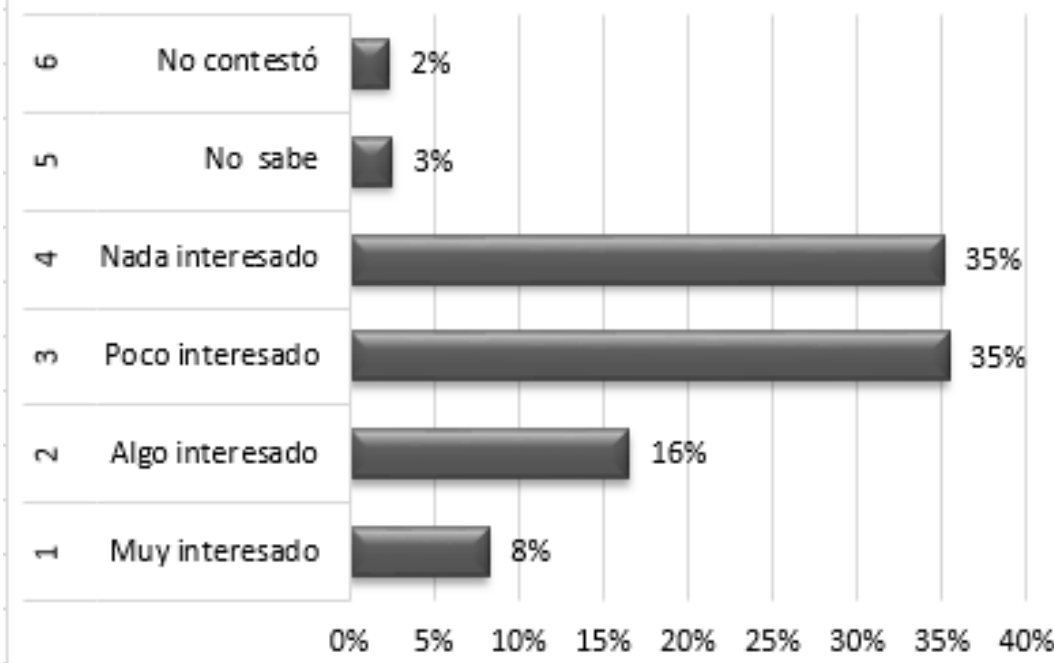

El abstencionismo es un fenómeno muy complejo, el cual engloba el descontento ciudadano y la anomalía institucional imperante en los espacios de decisión pública. Los valores cívicos de responsabilidad con el interés público son los que deben prevalecer en un estado democrático, la tendencia actual ha sido el disminuir el respeto hacia las instituciones y menguar la credibilidad de los espacios de deliberación pública.

Actualmente hay un desprecio ciudadano hacia las instituciones políticas, una decepción del modelo democrático, un vacío espiritual y cívico, lo cual incide en una falta de participación y una apatía hacia cualquier asunto que tenga que ver con las cuestiones políticas. Se dejan las decisiones más importantes del Estado en manos de los demás y el ciudadano renuncia a la responsabilidad de asumir su libertad con las consecuencias que ello implica (López, 2013). Lo anterior expuesto avala el poco interés manifestado por los encuestados en relación con la política y la participación ciudadana, reflejada en gran medida por el bajo nivel de conocimiento de la fecha en que se realizarán las siguientes elecciones federales.

\section{Voto Electrónico}

$\mathrm{Al}$ enfocarse sobre la influencia de la tecnología en la confiabilidad del voto electrónico, 66\% manifestó que no sería confiable, (Gráfica 4). 
Gráfica 4. Influencia de la tecnología en la confiabilidad del voto electrónico

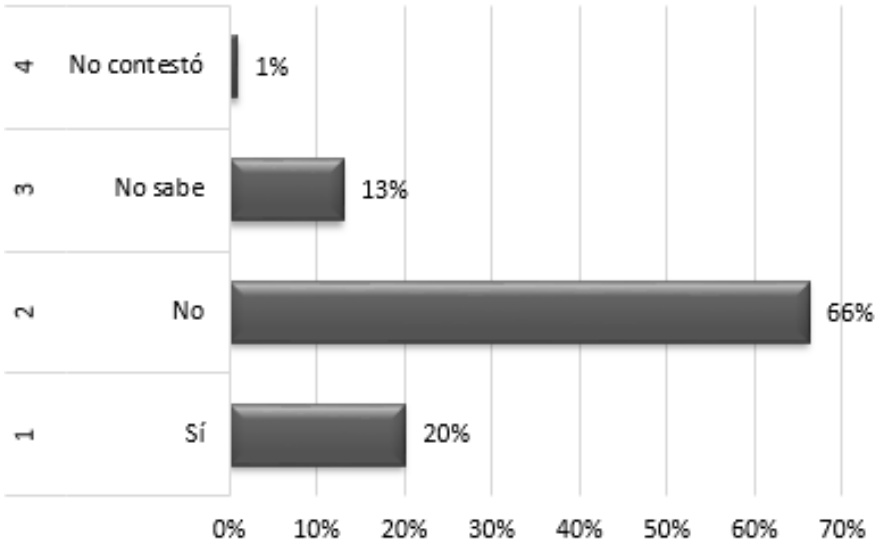

En el año 2012, se implementó un proyecto de voto electrónico en México, esto fue en las elecciones locales del estado de Jalisco. Se tuvieron algunos puntos de preocupación de este ejercicio, entre otros que como pruebas previas a la instalación de más de mil urnas electrónicas en dos distritos electorales y un municipio, las únicas pruebas de confiabilidad disponibles para ser analizadas públicamente fueron cinco simulacros (Wolf, 2012).

Al preguntar sobre cuál sería el tipo de tecnología más confiable para utilizarla en el voto electrónico, 63\% indicó que sería a través de un sufragio presencial por medio de urnas electrónicas instaladas en lugares habituales. Sólo 16\% consideró que se realizara a distancia por Internet, con un teléfono celular u otro dispositivo similar (Gráfica 5); lo cual, viene a corroborar el bajo nivel de confianza en los resultados electorales.

Gráfica 5. Tipo de tecnología para emitir el voto electrónico

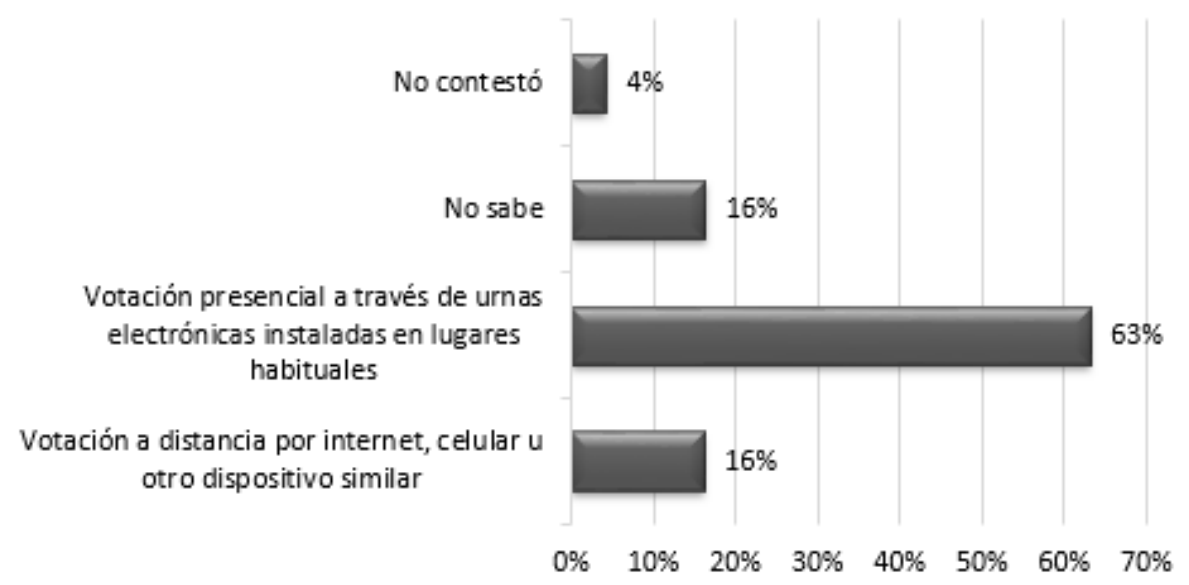


Una característica de los desarrolladores de software es la generación de errores en los mismos, además es difícil garantizar el hecho de tener una máxima seguridad en una computadora ante el acceso de extraños a la información contenida en ella (Wolf, 2012). Lo anterior va muy de la mano con la percepción de una baja confianza en la seguridad de la emisión del voto electrónico obtenido en este análisis.

El 39\% de los encuestados en el rango de edad que se está analizando (26 a 45 años), considera que el voto electrónico sería menos confiable en comparación con el sistema actual. Una minoría, 22\%, indicó que sería más confiable. Los datos que se muestran en la (Gráfica 6), es el resultado que algunos adultos de mayor edad, no son nativos de la tecnología, y tampoco no son usuarios en alta escala.

Gráfica 6. Grado de confiabilidad del voto electrónico

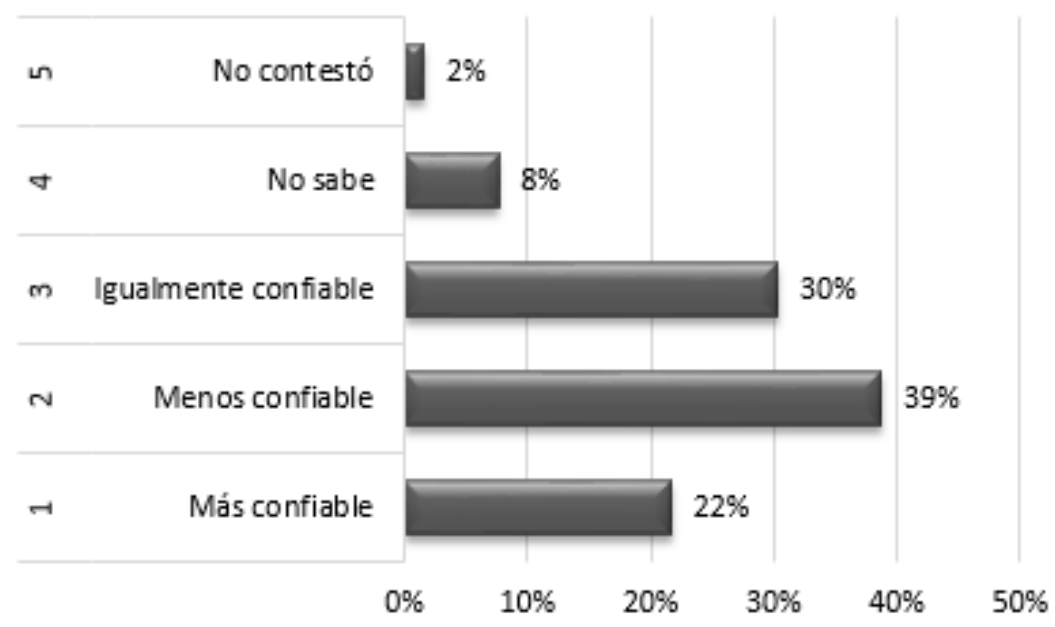

El avance de la tecnología informática indudablemente ha producido cambios sociales y del entorno, donde la democracia es otro de los campos a los que dicha revolución ha impactado a través de los medios electrónicos, en su elemento fundamental como mecanismos de expresión de la voluntad ciudadana, convergiendo en lo que se ha denominado voto electrónico. La velocidad de este cambio tecnológico ha propiciado, una nueva correlación entre la informática y el derecho político-electoral del voto, en lo que con seguridad constituirá el nuevo horizonte tecnológico en materia electoral. Si se considera la perspectiva de la Sociedad de la Información y el Conocimiento, la e-democracia o democracia electrónica se transforma, y de esta manera los derechos políticos se trastocan, los procesos electorales se envuelven en el flujo de información y la toma de decisiones se facilita, y así todo influye para la implementación del voto electrónico como una tendencia mundial irreversible anclada en las políticas públicas relacionadas con el gobierno electrónico, que pretenden materializar el anhelo de participación 
ciudadana a través de lo que podríamos definir como el ágora en su versión electrónica, en donde el elemento central es la tecnología basada en la informática (Téllez, 2010).

Sin embargo, la seguridad cibernética sigue siendo un tema a considerar como de vital importancia, para que la democracia en nuestro país se siga consolidando al respetarse la decisión de la ciudadanía. Las nuevas generaciones de votantes en nuestro país son nativos de la tecnología, para ellos la emisión del voto electrónico sería lo más natural, en contraparte, tenemos la capacitación que en cierto momento se pudiera requerir para los votantes de mayores rangos de edad, que no estén tan familiarizados con el uso de la tecnología de manera cotidiana. Un obstáculo en la emisión del voto electrónico podría ser el acceso a Internet en poblados remotos de nuestro país, por lo cual es válido el considerarse la combinación de los dos métodos de votación, el voto electrónico y el sistema de votación habitual. Inclusive la capacitación que se pudiera otorgar a los funcionarios de casilla, en caso de que se utilizaran las urnas electrónicas, podría ser más sencilla, clara y rápida, y en esa medida se podría generar una mayor motivación para la participación de los ciudadanos, al fungir como actores activos dentro de los procesos electorales a los cuales pudieran ser convocados.

En referencia al dinero público para el voto electrónico, 40\% dijo que generaría un ahorro, en cambio quienes opinan que se gastaría más, es reducido (Gráfica 7).

Gráfica 7. Costos por la emisión del voto electrónico

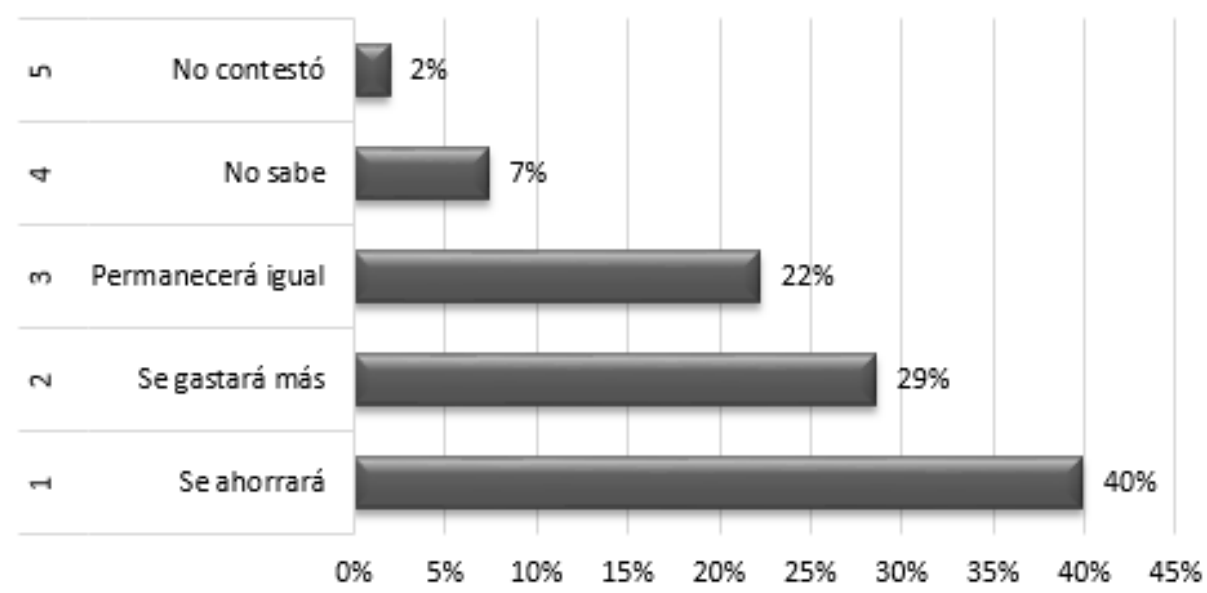

El peso fuerte de la población económicamente activa en nuestro país está comprendido entre los adultos jóvenes que son considerados en este análisis, donde, al tomar en cuenta la expectativa de vida de los mexicanos, tendrán una participación muy decisiva en los próximos procesos electorales 
que se darán en nuestro país, de ahí la importancia de considerarlos en este trabajo de investigación.

En la medida que el cambio en los procesos electorales se vaya dando acorde con los intereses de la población, la participación de los votantes podría darse en mayor medida; uno de los cambios importantes que se puede dar es el traslado del sistema tradicional de emisión de voto a la implementación del voto electrónico; ya sea considerando la implementación de las urnas electrónicas o bien contemplando la posibilidad de que el voto pueda ser emitido a distancia, a partir de cualquier dispositivo que tenga acceso a Internet.

Al considerar el hecho de si existe un nivel de urgencia en cuanto a usar el voto electrónico a nivel nacional, 27\% expresó en conjunto que es muy urgente, o algo urgente (Gráfica 8), lo cual es un porcentaje bajo para consideración de la urgencia de la implementación del voto electrónico en México.

Gráfica 8. Nivel de urgencia de implementación del voto electrónico

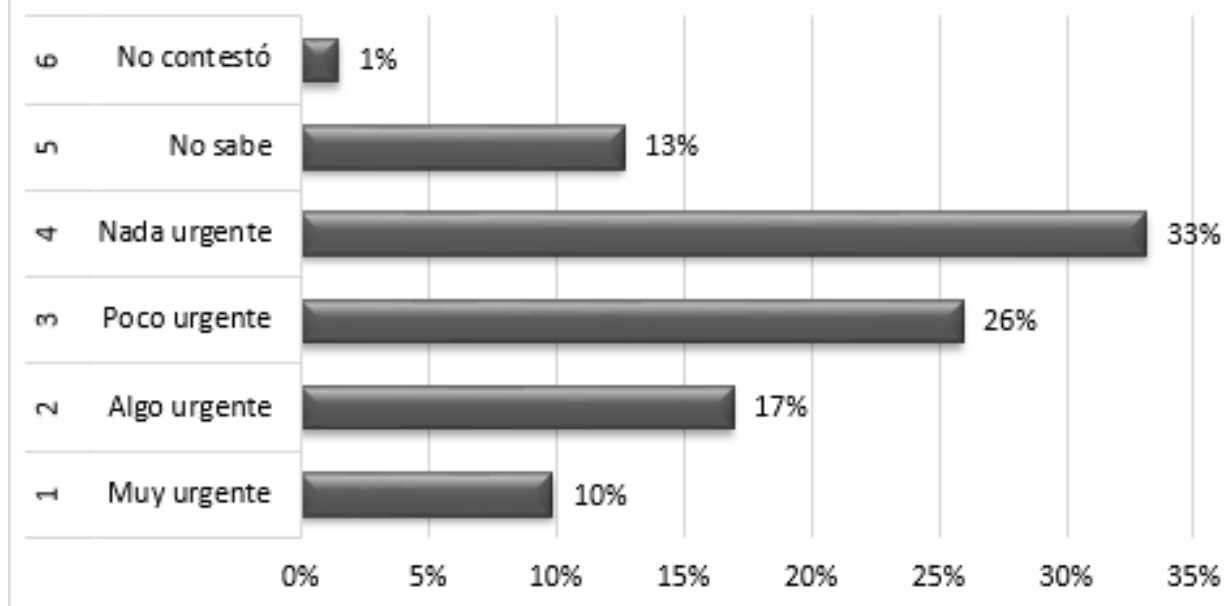

Algo fundamental en las diversas iniciativas de convergencia tecnológico-electorales vinculadas con el voto electrónico es que el aspecto técnico garantice de manera irrestricta el cumplimiento de los requisitos constitucionales y legales del voto público y más aún, que no deje lugar a suspicacia alguna en el procesamiento del fiel reflejo de la voluntad ciudadana expresada ahora por medios informáticos. En general, se puede señalar que para la implementación del voto electrónico se debería partir de tres condiciones básicas: 1 . Un nivel adecuado de aceptación social frente a las nuevas tecnologías aplicadas al ámbito electoral. 2. Factibilidad técnica que colme los requisitos constitucionales y legales en el ejercicio del sufragio público. 3. En especial, que resulte atractivo políticamente para los diversos actores en un contexto democrático (Téllez, 2010). Lo descrito 
coincide en gran medida con la percepción de los considerados en el estudio, donde manifiestan las ventajas del uso derivado del voto electrónico en cuanto a la obtención de resultados electorales, sin embargo el gran cuestionamiento es la percepción ciudadana en la seguridad con respecto a los resultados electorales que se obtengan.

\section{Modelo de Regresión Lineal Múltiple para indicadores de las variables}

Se obtuvo un modelo de regresión lineal múltiple para los indicadores elegidos para las variables, donde se consideró como dependiente al Grado de sufragios mediante el voto electrónico; el resto de los indicadores se tomaron como independientes. Este modelo sirve para predecir el grado de influencia que se tiene en el número de votos por parte de cada una de las demás variables consideradas como independientes. Se obtuvo una correlación significativa del modelo de regresión para las variables con las cuales se trabajó, estas se muestran en el (Cuadro 5); lo cual indica que sí hay influencia en la cantidad de sufragios emitidos por otros indicadores de las variables consideradas.

Cuadro 5. Resumen del modelo de regresión lineal múltiple

\begin{tabular}{cccc}
\hline $\mathrm{R}$ & R cuadrado & R cuadrado corregida & Error típico de la estimación \\
\hline 0.742 & 0.550 & 0.544 & 0.695 \\
\hline
\end{tabular}

De los ocho indicadores considerados como independientes para el modelo de regresión lineal múltiple, cinco resultaron significativos, los cuales son: Interés en la política (0.010), Tipo de tecnología para emitir voto electrónico (0.000), Grado de confiabilidad con el voto electrónico (0.000), Mayor confiabilidad con voto electrónico (0.000), Costo del voto electrónico (0.000) y Urgencia de implementación del voto electrónico (0.000). Por lo tanto estos niveles de significancia indican que son estos indicadores los que influyen en el Grado de votación emitido de manera electrónica.

La Gráfica 9, contiene la estadística de la lista nominal en México a1 13 de febrero de 2015, en la cual se muestra que de un total de 68,337,428 personas registradas en esta lista, 37,040,442 corresponden al rango de edad de 25 a 44 años, (54.2\%) del total de la lista; teniendo 9.2\% de diferencia con respecto al $45 \%$ del total de encuestados que arrojó la base de datos en el rango de 25 a 46 años que se consideraron para el análisis realizado.

El porcentaje por género del total de la lista nominal en el país al 13 de febrero de 2015 es cercano al 50\% para cada uno de los géneros, con 52\% de mujeres y 48\% de hombres según el INE (2015), los cuales son similares a los obtenidos en el rango de edad que fue analizado; sin embargo en el rango de edad considerado en este capítulo, los porcentajes con respecto a hombres y mujeres fueron inversos. 
Gráfica 9. Estadísticas por edad de la Lista Nominal en México, (INE, 2015)

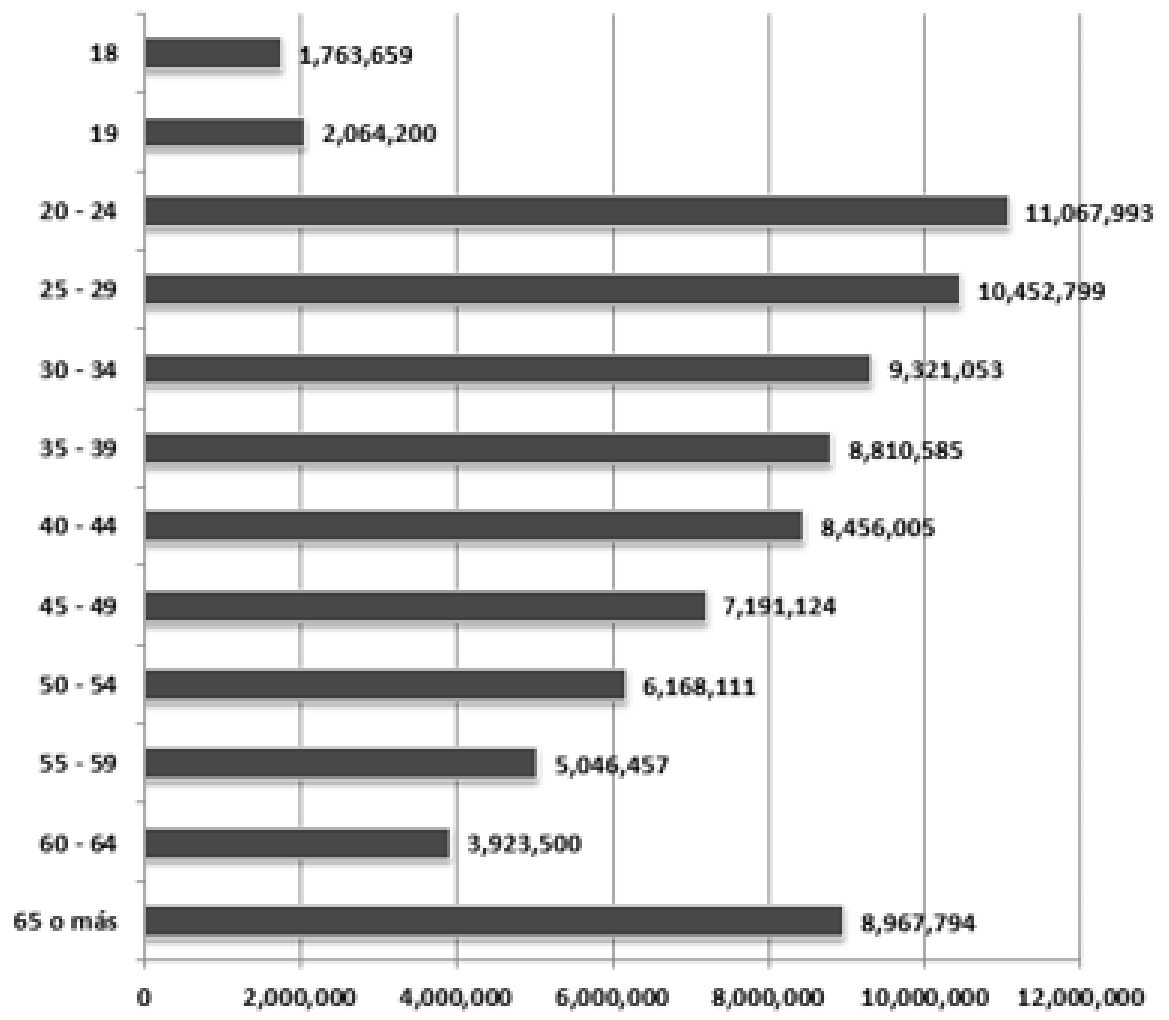

Una innovación en México fue resultado de una historia de desconfianza política, dando origen a la credencial para votar con fotografía con elementos de seguridad (Correa y Rocha, 2014); con lo cual se esperaba una mayor participación ciudadana en los procesos electorales. La mayor participación electoral en las elecciones federales de 2012 se da en los rangos de 40 a 79 años de edad, considerados en intervalos de 10 años, cada uno de ellos sobrepasó el $62.08 \%$ de la participación promedio de los electores en cada intervalo de edad. Es relevante considerar el hecho de que la participación de los jóvenes entre 18 y 19 años de edad, fue bastante alta en las elecciones federales de 2012, teniendo una participación cercana al 60\% del total de votantes en esa categoría.

\section{Conclusion}

Actualmente México se rige por una democracia consolidada en muchos ámbitos, incluidos los procesos electorales, nuestro país tiene una población en su mayoría joven (INEGI, 2015). Los jóvenes tienen un gran peso en cuanto a opinión y decisiones por la vía del voto, por lo que vienen a ser un factor fundamental en el desarrollo político-social en México. 
El interés en la política es muy bajo en el rango de población de adultos jóvenes, lo cual se debe considerar para la formulación de estrategias que conlleven a la difusión sobre las próximas elecciones, en caso contrario, podría verse reflejado un alto abstencionismo en este sector de la población, aun cuando la mayoría manifiesta que si votará o probablemente lo hará.

La generación comprendida en el rango de edad estudiado (26 a 45 años) utiliza los medios electrónicos como parte de su vida diaria, tanto personal como laboral, ya sea por el uso que se le da a las redes sociales, así como al Internet en general, debido a esto el tema del voto electrónico desde su percepción viene a ser de fundamental importancia.

La mayoría de los votantes adultos jóvenes manifiestan un bajo interés en los procesos electorales, por lo cual tal vez la utilización del voto electrónico, vendría a subsanar y posiblemente elevar el nivel de interés por estos procesos. Es de vital importancia dar a conocer a la ciudadanía en qué consiste el voto electrónico, así como manifestar cuáles serían sus ventajas potenciales frente al sistema tradicional de voto. En relación con el tema de la confiabilidad de sufragar de manera electrónica, la mayoría contesta que no es confiable; probablemente por la experiencia que se tiene sobre la seguridad de algunos sitios web en que ha sido vulnerada, pudiendo darse una asociación con la percepción que se tiene sobre este tema. En consecuencia, este rango de votantes manifiesta que para que la seguridad de la emisión del voto electrónico sea confiable, es mejor realizar el voto por medio de urnas electrónicas, situadas en los sitios habituales donde se realiza la votación tradicional. Se manifiesta además, un bajo nivel de confianza a emitir el voto desde sitios remotos a través de algún dispositivo móvil o computadora. El tema de seguridad y confianza reviste vital importancia para la adecuada implementación del voto electrónico, sobre todo para dar a la población en general la certeza de que su voto será respetado, independientemente de la manera como éste haya sido emitido. En términos generales un porcentaje ligeramente mayor a la mitad de estos votantes manifiesta que este tipo de voto electrónico puede ser igual o mayormente confiable que el sistema tradicional actual de emisión de voto.

Un alto porcentaje de los integrantes de este análisis manifiesta que el uso del voto electrónico haría que la gente votara en mayor medida. La facilidad que podría estar inmersa en este tipo de sufragio, podría estar más acorde a las actividades de la vida diaria actual.

El porcentaje mayor de este rango de votantes, manifiesta que se ahorrará gasto público en el uso del voto electrónico, considerando probablemente en gran medida que el número de personas involucradas en el proceso sería mayor, aun cuando en un inicio la inversión requerida para la implementación de este tipo de sufragio y lo que conlleva sería alta, aunque a 
la larga podría considerarse que pudiera generar un ahorro en las elecciones en nuestro país.

La percepción de la mayoría en este rango de votantes es que no es urgente la implementación del voto electrónico; sin embargo la determinación sobre la urgencia de la implementación de este por el instituto correspondiente, debe basarse en un concienzudo análisis de la situación actual del país. En la medida que se empiece a utilizar el voto electrónico y sean transparentes los resultados, se podrá ir elevando la confianza de los electores mediante su uso.

\section{References:}

1. Aguilar, R.J.A. (2014). Grandes expectativas: la democracia mexicana y sus descontentos. Revista Mexicana de Ciencias Polìticas y Sociales, UNAM. Año (49), No. (222). 19-50 pp.

2. Barrientos, DM.F. (2007). Dimensiones discursivas en torno al voto electrónico. Revista de Ciencia Política. Vol. (27), No. (1). 111-131 pp.

3. Correa, A.M.C. y R.D. Rocha (2014). Desmitificado el voto de los mexicanos en el exterior: retos, falta de voluntad y otras realidades. Perfiles Latinoamericanos. Vol. (22), No. (44). Recuperado de: http://www.scielo.org.mx/scielo.php?script=sci_arttext\&pid=S018876532014000200008

4. Hernández, M.A. (2008). La democracia mexicana, presa de una cultura política con rasgos autoritarios. Scielo, Revista mexicana de sociología. Vol. (70), No. (2). 261-303 pp.

5. Holzner, C.A. (2007). Voz y voto: participación política y calidad de la democracia en México. América Latina Hoy. No. (45). 69-87 pp.

6. INE, Instituto Nacional Electoral (2015). Estadísticas. Recuperado de:

http://www.ine.mx/archivos3/portal/historico/contenido/Estadisticas Lista_NominaN_y_Padron_Electoral/.Obtenido en: (22 febrero 2016)

7. INEGI, Instituto Nacional de Estadìstica y Geografìa (2015). Temas. Recuperado de: http://www3.inegi.org.mx/sistemas/temas. Obtenido en: (9 febrero 2016)

8. López, S.R. (2013). El abstencionismo como fenómeno político en la sociedad contemporánea. Revista Culturales. Vol. (1), No. (1). 53-68 pp.

9. Montoya, S.O. (2007). Aplicación del análisis factorial a la investigación de mercados. Caso de estudio. Scientia Et Technica . Vol. (13), No. (35). 281-286 pp.

10. Téllez, V.J. (2010). El Voto Electrónico. Temas Selectos de Derecho Electoral. TEPJF. 59 p. 
11. Tula, M.I. (2012). Democracia, elecciones y nuevas tecnologías. El voto electrónico. Revista Mexicana de Análisis Político y Administración Pública. Vol. (1), No. (2). 9-21 pp.

12. Velázquez, T.H. (2010). El voto electrónico en las elecciones de alcaldes en Venezuela. Espacios Públicos. No. (28). 102-112 pp.

13. Wolf, I.G.E. (2012). México, el voto electrónico y el 2012. Seguridad, Cultura de prevención para ti, UNAM. No. (14). Recuperado de:http://revista.seguridad.unam.mx/numero-14/m\%C3\%A9xico-elvoto-electr\%C3\%B3nico-y-el-2012. Obtenido en: (11 mayo 2016). 